\title{
Estudio del Plano Oclusal de una Población Chilena según los Cefalogramas de Steiner, Ricketts y Delaire
}

\author{
Study of the Occlusal Plane in a Chilean Population Using \\ the Cephalometric Analysis of Steiner, Ricketts and Delaire
}

\author{
Natalia Mora Figueroa*; Rolando Schulz Rosales*; Bárbara Cerda Peralta ${ }^{* * *}$; Macarena Rivera Rothgaenger ${ }^{\star * *}$; \\ Jimena López Garrido*; Valentina Martínez Arriagada* \& Fernando Romo Ormazábal ${ }^{* * *}$
}

MORA, F. N.; SCHULZ, R. R.; CERDA, P. B.; RIVERA, R. M.; LÓPEZ, G. J.; MARTÍNEZ, A. V. \& ROMO, O. F. Estudio del plano oclusal de una población chilena según los cefalogramas de steiner, ricketts y delaire. Int. J. Odontostomat., 9(3):379-384, 2015.

RESUMEN: El propósito de esta investigación fue comparar la ubicación del Plano Oclusal (PO) en una población eugnásica chilena mediante el análisis cefalométrico de Steiner, Ricketts y Delaire. Estudio transversal. Se analizaron 96 telerradiografías digitales de adultos jóvenes chilenos eugnásicos (47 hombres y 49 mujeres) entre 18 y 35 años. En las telerradiografías de determinó la ubicación del PO de acuerdo a los parámetros cefalométricos de Ricketts, Steiner y Delaire. Todos los trazados cefalométricos fueron realizados utilizando el software Adobe® Photoshop® CC, versión 14.2.1. Para las mediciones angulares correspondientes a los análisis de Steiner y Ricketts se utilizó el software Screen Protractor version 4.0@ 2006. Para las mediciones milimétricas correspondientes al análisis de Delaire, se utilizó el software Screen Calipers versión 4.0@ 2006. Al aplicar el análisis de Steiner en la muestra se obtuvo un promedio de $17,16^{\circ}$ para el ángulo formado entre el plano Silla-Nasion y el PO (norma $14,5 \pm 3^{\circ}$ ). Al utilizar el análisis de Ricketts, el ángulo promedio formado por el plano de Frankfurt y el PO fue $6,98^{\circ}$ (norma $7,5 \pm 2^{\circ}$ ) Según el análisis de Delaire, la distancia en milímetros desde el $\mathrm{PO}$ al punto medio entre las cúspides vestibulares del primer y segundo premolar inferior fue 1,76 mm (referencia teórica $0 \pm 1 \mathrm{~mm}$ ). El promedio de la angulación del PO se encuentra dentro del rango de normalidad según la norma de Steiner y Ricketts y el promedio de la distancia medida en milímetros del PO al punto medio entre las cúspides vestibulares del primer y segundo premolar inferior difiere en $1,76 \mathrm{~mm}$ de la referencia teórica del análisis cefalométrico de Delaire. Se recomienda realizar nuevos estudios que permitan definir las normas cefalométricas apropiadas para la poblacion chilena, lo que podría mejorar tanto diagnóstico como planes de tratamiento en diversas áreas de la odontología.

PALABRAS CLAVE: plano oclusal, cefalometría, eugnásico, chileno.

\section{INTRODUCCIÓN}

El plano oclusal (PO) es el "plano promedio formado por las superficies incisales y oclusales de los dientes" (The glossary of prosthodontic terms, 2005). Se ha descrito que ligeras variaciones en la configuración del plano oclusal alteran la armonía del sistema estomatognático (Ash \& Ramfjord, 1995; Chan, 2007), por lo cual su determinación resulta esencial en la práctica odontológica, en particular en procedimientos que requieran análisis y/o intervención oclusal.

Existen diversos métodos para establecer la ubicación del PO, sin embargo la mayoría resultan ser poco objetivos. La cefalometría es una herramienta de medición estandarizada y objetiva ampliamente utilizada en diversas áreas de la odontología, que puede resultar útil en la determinación del PO. El análisis cefalométrico establece relaciones horizontales y verticales entre los componentes del macizo craneofacial, determinando rangos de referencia para una población particular (Barahona \& Benavides, 2006). De este modo, permite detectar cualquier diferencia entre las relaciones dentofaciales de un individuo en particular y lo que se esperaría encontrar según su grupo étnico o racial.

\footnotetext{
Licenciada en Odontología, Clínica Integral del Adulto, Facultad de Odontología, Universidad de Chile, Santiago, Chile.

" Profesor Asistente, Clínica Integral del Adulto, Facultad de Odontología, Universidad de Chile, Santiago, Chile.

*** Instructor, Clínica Integral del Adulto, Facultad de Odontología, Universidad de Chile, Santiago, Chile.

Profesor Titular, Departamento de Prótesis, Facultad de Odontología, Universidad de Chile, Santiago, Chile.
} 
Desde el surgimiento de la cefalometría radiológica en 1934, diversos autores han propuesto definiciones para el trazado del PO relacionándolo con estructuras anatómicas en el cráneo. Steiner (1953) trazó el PO a través de la línea de intercuspidación de los primeros molares y un punto equidistante de los bordes incisales de los incisivos superiores e inferiores. Ricketts et al. (1961) definieron el PO como la línea trazada desde la cúspide mesiobucal del primer molar permanente mandibular y la cúspide del primer premolar mandibular. Delaire (1978) definió el PO como una línea tangente a la superficie oclusal de los premolares. Plantea que en una situación ideal el plano oclusal intersecta con los planos maxilar y mandibular en un mismo punto (Fig. 1).
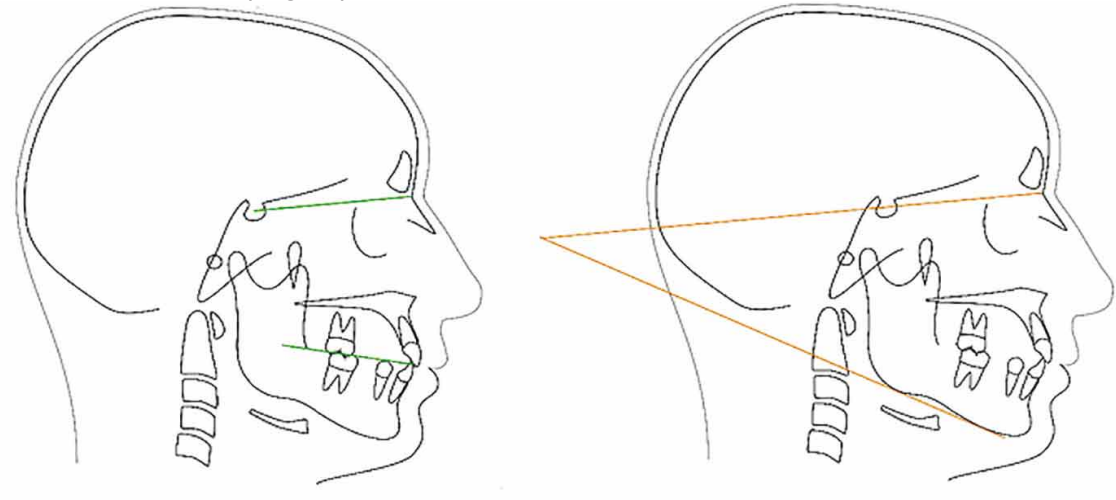

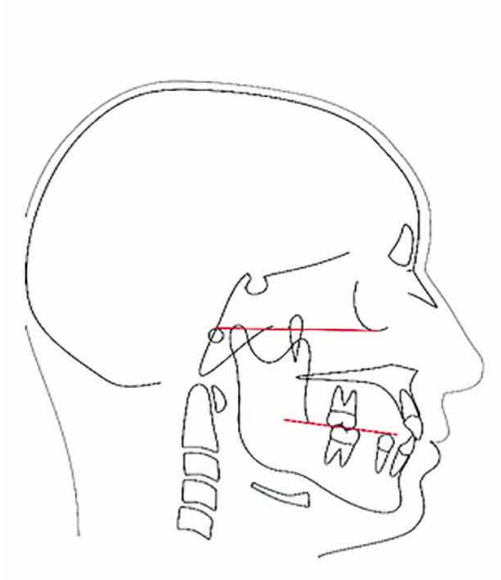

Ricketts
Steiner

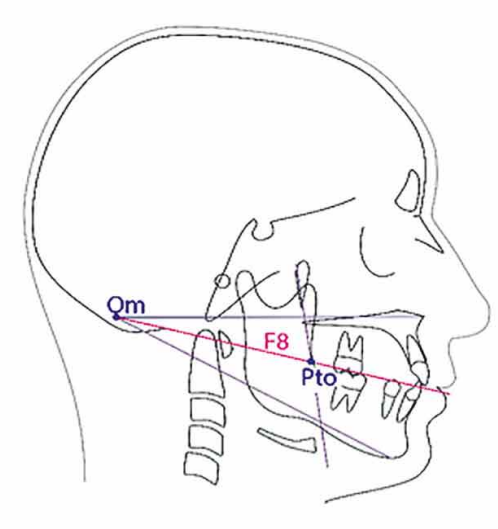

Delaire

Fig. 1. a) Plano Silla Nasion - Plano Oclusal (Steiner); b) Ángulo cráneomandibular (Steiner); c) Plano de Frankfurt - Plano oclusal (Ricketts) y d) Plano oclusal (Delaire).

La cefalometría en Chile se inició en 1962 utilizando mayoritariamente análisis cefalométricos de origen europeo. Sin embargo, Hoffens \& San Pedro (1973) evidenciaron que las medidas cefalométricas de los análisis clásicos no concordaban con los casos chilenos. Venegas (1980), Schulz et al. (1985), Julia Garau (1985) y Sandoval et al. (2011) estudiaron poblaciones chilenas infantiles. Coincidieron en que los chilenos poseen un conjunto de medidas que les son propias como población y señalaron la necesidad del uso de parámetros cefalométricos que se adecúen a la realidad étnica de Chile.
En esta investigación se determinó la ubicación del plano oclusal de una población eugnásica chilena a fin de comparar el grado de concordancia existente con las normas de los análisis cefalométricos de Steiner, Ricketts y Delaire.

\section{MATERIAL Y MÉTODO}

Estudio transversal. Se analizaron 96 telerradiografías de perfil digitales de jóvenes chilenos (49 mujeres y 47 hombres) entre 18 y 35 años de edad.

Fueron incluidos sujetos eugnásicos con dentición natural completa, clase esqueletal I o clase II leve (sin necesidad de tratamiento), presencia de acople anterior y articulación témporomandibular con rangos de movilidad normales, sin síntomas asociados. Se excluyeron aquellos individuos disgnásicos, con cualquier patología que haya producido una alteración del crecimiento vertical (patologías como: mordida abierta, mordida cubierta y patología articular degenerativa), clase esqueletal II o III evidentes, sometidos a un tratamiento de ortodoncia (previo o en curso), antecedentes de cirugía ortognática u otra cirugía que altere la morfología facial, historial de traumatismos craneofaciales, malos hábitos orales (respiración oral, interposición lingual, labial o de objetos) y aquellos con apiñamiento dentario severo (Índice IOTN mayor a 2) (Johansson \& Follin, 2009).

Cada participante del estudio firmó un consentimiento informado aprobado por el Co- 
mité de Ética de la Facultad de Odontología de la Universidad de Chile, que explicó en detalle todos los procedimientos que se practicaron y las implicancias que podrían tener para cada uno de ellos. Los exámenes radiográficos fueron realizados bajo estricto respeto de las normas de radioprotección.

Se empleó un equipo imagenológico digital (Sirona ${ }^{\circledR}$ Orthophos XG Plus ${ }^{\circledR}$ ) según las indicaciones del fabricante. Las piezas dentarias estaban en posición de oclusión (MIC) y los labios en reposo. La posición de la cabeza de cada sujeto se encontraba con el plano de Frankfurt paralelo al piso. Los brazos relajados y las piernas levemente separadas. Se pidió al paciente que deglutiera y después de unos segundos (luego de entrar en MIC) se tomó la radiografía.

Los trazados cefalométricos fueron realizados por un operador utilizando Adobe $\AA$ Photoshop $®$ CC versión 14.2.1. Las mediciones fueron realizadas utilizando los softwares Screen Calipers versión 4.0@ 2006 y Screen Protractor versión 4.0@ 2006.

Análisis de Steiner. Se midió la angulación existente entre el plano Silla-Nasion y el PO definido por Steiner, cuya norma es $14,5 \pm 3^{\circ}$ (Fig. 1). Se midió el ángulo cráneomandibular formado entre el plano Silla-Nasion y el plano Gonion-Gnation definido por Steiner, cuya norma es $32^{\circ}$ (Fig. 1).

Análisis de Ricketts. Se midió la angulación existente entre el plano de Frankfurt y el PO definido por Ricketts (Fig. 1). Para dicho ángulo, Ricketts determinó como norma $7,5^{\circ}$.
Existe muy poca información sobre los rangos de tolerancia para puntos cefalométricos y ángulos en el análisis de Ricketts. En 1996, Águila investigó la validación y precisión en la identificación de éstos, determinando que el error de método es $1 \mathrm{~mm}$ para el promedio del punto cefalométrico y dos grados para el promedio de los ángulos. Considerando los resultados de Águila, el rango de tolerancia utilizado en este estudio para el ángulo formado entre el plano de Frankfurt y el PO fue $7,5 \pm 2^{\circ}$.

Análisis de Delaire. El análisis de Delaire se compone de una serie de trazados que evidencian la existencia de alteraciones en el macizo craneofacial de un individuo y la posible causa de esta alteración. Para éste análisis es imprescindible una telerradiografía de perfil de cráneo completo, sin embargo la gran mayoría de los equipos utilizados no entregan ese tipo de imagen, razón por la cual se modificó el esquema de trazado sin transgredir las bases de la filosofía de Delaire (Fig. 2):
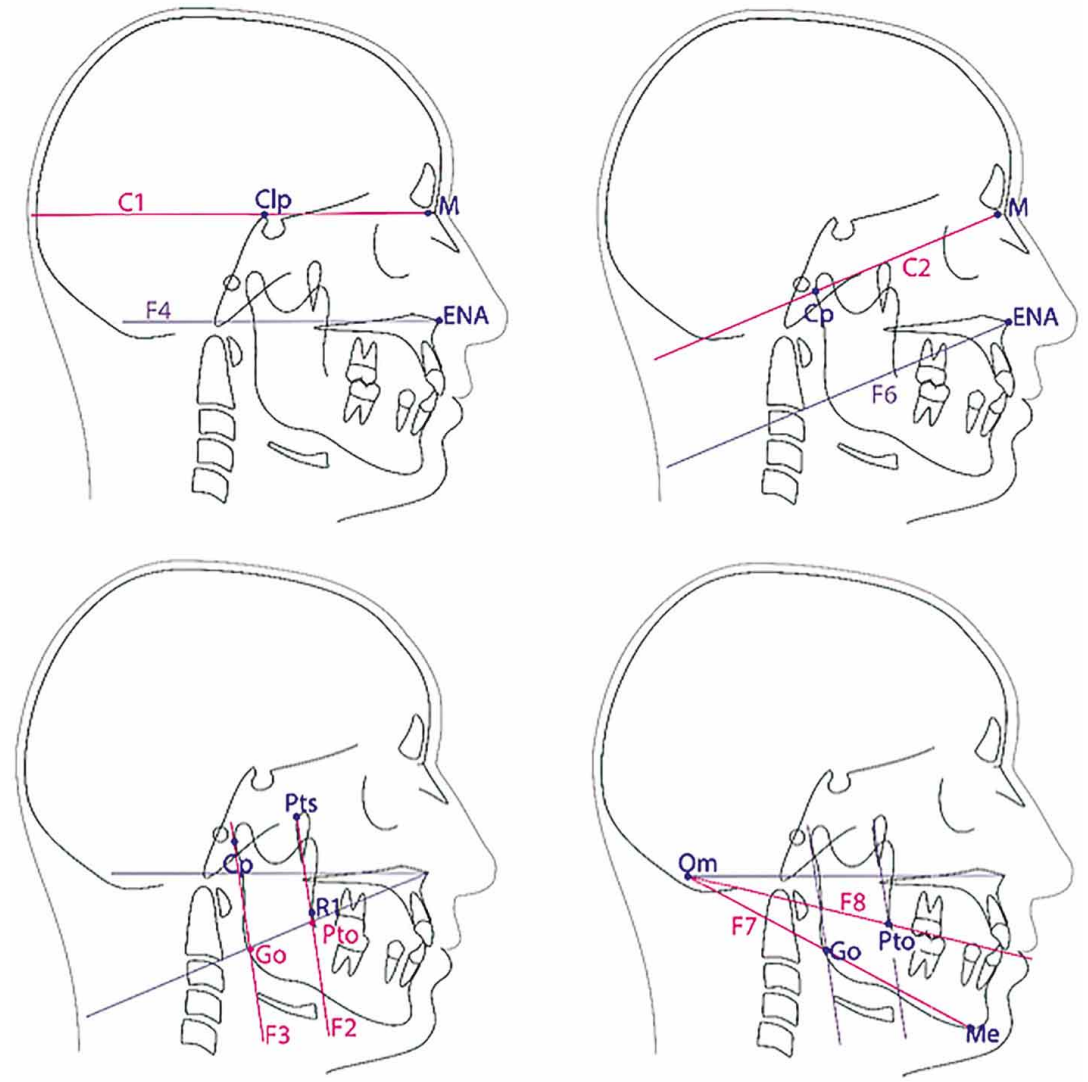

Fig. 2 Trazado modificado de Delaire et. al. (1981).

C1: Se traza desde el punto M hasta el punto Clp.

F4 (Plano Maxilar): Se traza paralela a C1 pasando por ENA.

C2: Línea trazada desde el punto $\mathrm{M}$ hasta el punto $\mathrm{Cp}$.

F6: Línea paralela a C2 a partir de ENA.

F2: Se traza una recta desde el punto R1 al punto Pts.

F3: Línea paralela a F2 pasando por Cp.

F2 y F3 al intersectar con F6 definen el punto Pto y Go, respectivamente.

F7 (Plano Mandibular): Se traza una línea desde el punto Me hasta Go y se extiende la recta hasta intersectar F4 para definir punto Om.

F8 (Plano Oclusal): Trazado desde el punto Pto al punto Om. 
Luego de realizar el trazado, se midió la distancia vertical en milímetros $(\mathrm{mm})$ desde la línea F8, en forma perpendicular, hasta el punto medio entre las cúspides vestibulares del primer y segundo premolar inferior. El error de método determinado por Águila (1996) en términos métricos corresponde a 1 $\mathrm{mm}$, consecuentemente el rango de tolerancia que se utilizó fue $0 \pm 1 \mathrm{~mm}$.

Los datos obtenidos en el estudio fueron analizados utilizando el Software Stata $12 \circledast$ (de Stata Corporation L.P.) Las medidas de resumen se exhiben en la Tabla I.

La normalidad de la muestra fue determinada con el test Shapiro-Wilk ( $p>0,05$ para los tres análisis utilizados).

\section{RESULTADOS}

Para el análisis de Steiner, al medir el ángulo formado entre el Plano SN y el PO se obtuvo un promedio de $17,16^{\circ}$, siendo la norma $14,5 \pm 3^{\circ}$. Al medir el ángulo craneomandibular el promedio obtenido fue $32,65^{\circ}$ siendo la norma $32^{\circ}$.

Para el análisis de Ricketts, en promedio, el ángulo formado entre el Plano de Frankfurt y el PO en la muestra es $6,98^{\circ}$ (norma: $7,5 \pm 2^{\circ}$ ).

Para el análisis de Delaire, la distancia en milímetros desde el PO al punto medio entre las cúspides bucales del primer y segundo premolar mandibular obtenida de la muestra es $1,76 \mathrm{~mm}$. Se consideró como rango de aceptación $0 \pm 1 \mathrm{~mm}$.

Tabla I. Resultados obtenidos al analizar as variables cefalométricas en 96 telerradiografías de perfil digitales de jóvenes chilenos entre 18 y 35 años de edad.

\begin{tabular}{lcccc}
\hline Variable & Promedio & Desviación Estándar & Min. & Max. \\
\hline PO Steiner - P.Silla/Nasion & $17,16^{\circ}$ & $4,07^{\circ}$ & $7,36^{\circ}$ & $26,32^{\circ}$ \\
Hombre & $15,73^{\circ}$ & $3,94^{\circ}$ & $7,36^{\circ}$ & $25,98^{\circ}$ \\
Mujer & $18,59^{\circ}$ & $3,70^{\circ}$ & $10,68^{\circ}$ & $26,32^{\circ}$ \\
Ángulo-cráneomandibular & $32,65^{\circ}$ & $6,15^{\circ}$ & $20,10^{\circ}$ & $47,80^{\circ}$ \\
Mujeres & $34,61^{\circ}$ & $5,59^{\circ}$ & $23,70^{\circ}$ & $47,80^{\circ}$ \\
Hombres & $30,69^{\circ}$ & $6,12^{\circ}$ & $20,10^{\circ}$ & $45,40^{\circ}$ \\
PO Ricketts - P.Frankfurt (mm) & 6,98 & 3,87 & $-2,73$ & 16,85 \\
Hombre & 6,12 & $3,79^{\circ}$ & $-2,73$ & 12,00 \\
Mujer & 7,84 & $3,80^{\circ}$ & 0,99 & 16,85 \\
PO Delaire - Distancia a premolares (mm) & 1,76 & 3,07 & $-4,14$ & 9,43 \\
Hombre & 2,12 & 3,39 & $-4,14$ & 9,00 \\
Mujer & 1,41 & 2,70 & $-3,16$ & 9,43 \\
\hline
\end{tabular}

\section{DISCUSIÓN}

Los análisis cefalométricos evaluados en este estudio son los más utilizados por los clínicos en Chile. Considerando los valores de error de método definidos por Águila, existen mínimas variaciones entre la ubicación del plano oclusal de la población estudiada y la norma determinada por Steiner y Ricketts, más notorias en el análisis de Delaire, lo que evidencia que los chilenos poseen características propias, definidas por su genética y epigenética.

Análisis de Steiner. En el año 1970, Gamm \& Giannely realizaron un estudio en Boston el que utilizaron una muestra de 77 niños entre 8 y 13 años con oclusión normal, perfiles rectos y armoniosos. Para el ángulo formado entre el PO y el plano Silla-Nasion, obtuvieron $17,3 \pm 1,2^{\circ}$, mientras que para el ángulo cráneomandibular (GoGn-SN) obtuvieron $32,8 \pm 3,5^{\circ}$. Ambos resultados son concordantes con los obtenidos en esta investigación.

Astorga Hollstein (1993) obtuvo $13,73^{\circ}$ al medir el ángulo formado entre el Plano Silla-Nasion y el Plano Oclusal, y $29,38^{\circ}$ para el ángulo cráneomandibular en una muestra de 34 individuos adultos jóvenes chilenos entre 18 y 30 años. Una diferencia en relacion a esta investigación es que Astorga Hollstein realizó el análisis cefalométrico de forma manual. Si bien los resultados de esta investigación y los obtenidos por el autor concuerdan con la norma definida por Steiner, 
ambos presentan distintos promedios. Esto puede deberse a que las muestras difieren en tamaño, siendo mayor la cantidad de individuos en el presente estudio.

Análisis de Ricketts. En 1950 Ricketts estableció la norma para el ángulo formado entre el $\mathrm{PO}$ y el plano de Frankfurt al estudiar a un grupo de 50 individuos entre 4 y 40 años, con oclusión normal o leve maloclusión, incluyendo individuos clase I, II y III de Angle, lo que explicaría las diferencias encontradas en este estudio respecto a la referencia determinada por Ricketts.

En Chile, Garau (1985) comparó 51 individuos entre 8 y 15 años con las normas cefalométricas del análisis frontal de Ricketts. Encontró diferencias entre las medidas de la muestra y las normas de Ricketts, concluyendo que los chilenos poseen un conjunto de medidas que le son propias como población.

Análisis de Delaire. El análisis de Delaire fue sometido a modificaciones en este estudio con el fin de simplificar su aplicación. Frugone \& Pantoja (2008) utilizó una muestra de 78 pacientes con necesidad de rehabilitación. De esta muestra, 26 presentaban desgaste dentario severo simétrico y 52 no presentaban desgaste dentario, definidos como el grupo control. Al trazar el plano oclusal en el grupo control se encontraron diferencias respecto al plano oclusal teórico menores a $1 \mathrm{~mm}$. Se concluyó que la mayoría de los individuos sanos sin desgaste dentario presentan un plano oclusal real altamente relacionado con el PO teórico definido por Delaire. En 2013, Antonarakis et al. utilizaron el análisis de Delaire para examinar a 30 pacientes suizos, clase I oclusal entre 18 y 61 años. Concluyeron que el plano oclusal teórico de Delaire es raramente coincidente en pacientes clase I oclusal sin desarmonías dentofaciales.

La utilidad clínica de los parámetros cefalométricos es diferente para cada especialidad odontológica, esto responde a la diferencia entre las patologías tratadas por cada especialidad y la diferencia en los tratamientos necesarios para su resolución. Por lo tanto, los rangos de variación, más allá de la norma estadística, tienen un valor clínico según la utilidad prestada al profesional.

Finalmente, se concluye que el promedio de la angulación del PO se encuentra dentro del rango de normalidad según la norma de Steiner y Ricketts y que el promedio de la distancia medida en milímetros del PO de la población estudiada difiere en $1,76 \mathrm{~mm}$ de la referencia teórica del análisis cefalométrico de Delaire.

AGRADECIMIENTOS: AI Dr. Rodrigo Bravo Ahumada por su desinteresada colaboración en este proyecto.

MORA, F. N.; SCHULZ, R. R.; CERDA, P. B.; RIVERA, R. M.; LÓPEZ, G. J.; MARTíNEZ, A. V. \& ROMO, O. F. Study of the occlusal plane in a Chilean population using the cephalometric analysis of Steiner, Ricketts and Delaire. Int. J. Odontostomat., 9(3):379-384, 2015.

ABSTRACT: The aim of this study was to compare the location of the occlusal plane (OP) in a eugnathic Chilean population using Steiner, Ricketts, and Delaire cephalometric analysis. A cross-sectional study was carried out and ninetysix digital cephalometric radiographs from 47 male and 49 female young adults between ages 18 and 35 were analyzed to determine the location of the OP according to Steiner, Ricketts and Delaire cephalometric parameters. Cephalometric tracings were obtained with Adobe ${ }^{\circledR}$ Photoshop ${ }^{\circledR}$ CC, version 14.2.1 for all three analysis. Screen Calipers version $4.0 \odot 2006$ software was used to measure the distance in millimeters from the OP to a midpoint between the first and second inferior premolars cusps for Delaire analysis. For Steiner and Ricketts angle measurements, software Screen Protractor version 4.0@ 2006 was used. Mean and Standard Deviation Tests for Steiner analysis showed that the mean angle between SellaNasion plane and OP was $17.16^{\circ}$ (cephalometric norm $14.5 \pm 3^{\circ}$ ). The mean angle between Frankfurt plane and OP was $6.98^{\circ}$ (cephalometric norm $7.5 \pm 2^{\circ}$ ) for Ricketts analysis. In Delaire analysis, the distance in millimeters from the OP to a midpoint between the first and second inferior premolars cusps obtained was $1.76 \mathrm{~mm}$, which differs from the theoretical reference $(0 \pm 1 \mathrm{~mm})$. The mean angle of the occlusal plane obtained is within the average standards for Steiner and Ricketts analysis. The mean distance in millimeters obtained from the OP to a midpoint between the first and second inferior premolars cusps in this study differs from the theoretical reference reported in Delaire analysis. Further studies are recommended to study more appropriate cephalometric norms for the Chilean population that would improve diagnosis and treatment plans in different areas of the dentistry field.

KEY WORDS: occlusal plane, cephalometry, eugnathic, Chilean. 
MORA, F. N.; SCHULZ, R. R.; CERDA, P. B.; RIVERA, R. M.; LÓPEZ, G. J.; MARTíNEZ, A. V. \& ROMO, O. F. Estudio del plano oclusal de una población chilena según los cefalogramas de steiner, ricketts y delaire. Int. J. Odontostomat., 9(3):379-384, 2015.

\section{REFERENCIAS BIBLIOGRÁFICAS}

Águila, J. Manual de Cefalometría. Caracas, Editorial Actualidades Médico Odontológicas Latinoamericanas, 1996. pp.3-17.

Antonarakis, G. S.; Kiliaridis, S. \& Scolozzi, P. Orientation of the occlusal plane in a Class I adult population. Oral Surg. Oral Med. Oral Pathol. Oral Radiol., 116(1):35-40, 2013.

Ash, M. M. \& Ramfjord, S. P. Occlusion. $4^{\text {th }}$ ed. Philadelphia, W. B. Saunders, 1995. pp.1-29.

Astorga Hollstein, I. D. Estudio comparativo de los análisis cefalométricos de Downs y Steiner en una muestra de individuos adulto joven chilenos. Tesis para optar al título de cirujano-dentista. Santiago de Chile, Facultad de Odontología, Universidad de Chile, 1993.

Barahona, C. J. B. \& Benavides, S. J. Principales análisis cefalométricos utilizados para el diagnóstico ortodóntico. Rev. Cient. Odontol., 2(1):11-27, 2006.

Chan, C. A. A Review of the Clinical Significance of the Occlusal Plane: Its Variation and Effect on Head Posture. Int. Coll. Craniomandib. Orthop. Anthol., 8:1-63, 2007.

Delaire, J.; Schendel, S. A. \& Tulasne, J. F. An architectural and structural craniofacial analysis: a new lateral cephalometric analysis. Oral Surg. Oral Med. Oral Pathol., 52(3):226-38, 1981.

Frufone, Z. R. \& Pantoja Parada, R. Características craneofaciales en pacientes con desgaste dentario severo. Rev. Fac. Odontol. Univ. Antioq., 21(2):142-9, 2010.

Gamm, S. H. \& Gianelly, A. A. Polygonic interpretation of the Steiner analysis. Am. J. Orthod., 58(5):479-85, 1970.

Garau, M. C. Análisis cefalométrico frontal de una muestra de individuos chilenos normales. Trabajo de investigación. Curso de especialización en Ortopedia Dentomaxilar. Escuela de Graduados. Santiago, Universidad de Chile, 1985.

Hoffens, E. \& San Pedro, J. Análisis cefalométrico de eugnásicos chilenos en telerradiografía de perfil. Odontol. Chil., 21(108):32-5, 1973.

Johansson, A. M. \& Follin, M. E. Evaluation of the Dental Health Component, of the Index of Orthodontic Treatment Need, by Swedish orthodontists. Eur. J. Orthod., 31(2):184-8, 2009.

Julia Garau, M. C. Análisis cefalométrico frontal de una muestra de individuos chilenos normales. Santiago de Chile, Facultad de Odontología. Universidad de Chile, 1985.
Ricketts, R. M. Variations of the temporomandibular joint as revealed by cephalometric laminagraphy. Am. J. Orthod., 36(12):877-98, 1950.

Sandoval, P.; García, N.; Sanhueza, A.; Romero, A. \& Reveco, R. Cephalometric measurements in lateral radiographs of five-year-old pre-schoolers in the city of Temuco. Int. J. Morphol., 29(4):1235-40, 2011.

Schulz, R.; Ramirez C., J. \& Ansoleaga I., A. Cefalograma de Schwarz en 30 individuos eugnásicos chilenos con dentadura temporal mixta definitiva. Rev. Chil. Ortod., 2(2):103-7, 1985.

Steiner, C. C. Cephalometrics for you and me. Am. J. Orthod., 39(10):729-55, 1953.

The glossary of prosthodontic terms. J. Prosthet. Dent., 94(1):10-92, 2005.

Venegas, A. Análisis cefalométrico en individuos eugnásicos seleccionados de una muestra escolar. Tesis para optar al título de cirujano-dentista. Santiago de Chile, Facultad de Odontología. Universidad de Chile, 1980.

Dirección para Correspondencia:

Natalia Mora Figueroa

Cirujana dentista

Universidad de Chile

CHILE

Email: natilymora@gmail.com

Recibido : 31-03-2015

Aceptado: 29-09-2015 